\title{
Age-Related Changes in Central Nervous System Phosphatidylserine Decarboxylase Activity
}

\author{
Gabriela A. Salvador, Frederico M. López, and Norma M. Giusto \\ Instituto de Investigaciones Bioquímicas, Universidad Nacional del Sur y Consejo Nacional de Investigaciones \\ Científicas y Técnicas, Argentina
}

A significant portion of brain phosphatidylethanolamine $(\mathrm{PE})$ is synthesized by a pathway involving the mitochondrial enzyme phosphatidylserine decarboxylase (PSDC), in a process by which phosphatidylserine (PS) is transferred from the endoplasmic reticulum to mitochondria. Aging changes the fatty acid composition of brain phospholipids, PS and PE being the most affected. The present study was carried out to determine PSDC activity in cerebral cortex (CC) and cerebellum (CRBL) mitochondrial fraction from adult (4-month-old) and aged (30month-old) rats and to compare these activities with that found in liver. To study the effect of 22:6n-3 content on the PSDC activity, PSs from different sources were prepared: rPS (from bovine retina, containing $36 \mathrm{~mol} \%$ of $22: 6 n-3)$; adPS (from adult rat CC microsomal membranes, with 25 mole \% 22:6n-3 content) and agPS (from aged rat CC microsomal membranes, with 21 mole \% 22:6n-3 content). For aged CC PSDC, the preferred substrate was agPS (the physiological substrate for aged animals), whereas in adult CC PSDC the substrate preference was inverse (rPS $>$ adPS $>$ agPS). Furthermore, CRBL PSDC does not show any substrate preference based on 22:6n-3 content. CRBL PSDC activity in aged membranes using agPS as substrate is lower than PSDC activity in adult membranes in the presence of adPS. These results indicate that under physiological conditions, cerebellar PSDC is inhibited during aging. Liver PSDC activity showed the same substrate preference in adult and aged rats as adult CC PSDC. These findings lead us to conclude that PSDC activity has a differential tissue-dependent substrate preference characteristic of the aging process. $\odot 2002$ Wiley-Liss, Inc.

Key words: PSDC; aging; phosphotidylserine

Aging has been defined as the progressive accumulation of changes that are either related to or responsible for the ever-increasing susceptibility to diseases and death that accompany advancing age (Harman, 1991). The molecular bases of aging are still unknown, although many theories support the idea that age-related deterioration of physiological functions is caused by the modification of cellular constituents (Giusto et al., 1992; Yen et al., 1994;
Lopez et al., 1995). Lipids are one of such constituents that undergo changes during aging. Phospholipids as an integral part of membranes play an important role in the regulation of cell membrane function and any modification in their metabolism may therefore influence cellular function. Many membrane-bound enzymes and transport systems are affected by alterations in the properties of the lipid bilayer (Lynch, 1980; Stubbs and Smith, 1984).

It has been reported that brain aging is accompanied by changes in the activities of several enzymes that modify the polar portion of phospholipid moiety such as: baseexchange enzymes, phospholipase D, phosphatidylethanolamine N-methyltransferase and phosphatidate phosphohydrolase, among others (Ilincheta de Boschero et al., 2000a; Ilincheta de Boschero et al., 2000b; Pasquaré et al., 2001; Salvador et al., 2002). Taking into account these findings, it can thus be concluded that age affects some of the alternative pathways for phospholipid synthesis, indicating the presence of compensatory mechanisms that provide a pool of phospholipid classes for the maintenance of cellular membrane lipid composition in the CNS.

In the brain, phosphatidylethanolamine $(\mathrm{PE})$ can be synthesized from free ethanolamine through the cytidine phosphoethanolamine pathway (Kennedy and Weiss,

Abbreviations used: adPS, phosphatidylserine from adult cerebral cortex microsomes; agPS, phosphatidylserine from aged cerebral cortex microsomes; CM, crude mitochondrial membranes; CNS, central nervous system; CRBL, cerebellum; CC, cerebral cortex; PE, phosphatidylethanolamine; PS, phosphatidylserine; PSDC, phosphatidylserine decarboxylase, rPS, phosphatidylserine from bovine retina; TLC, thin-layer chromatography.

Contract grant sponsor: Consejo Nacional de Investigaciones Cientificas y Técnicas (CONICET); Contract grant sponsor: the Secretaría General de Ciencia y Tecnología, Universidad Nacional del Sur; Contract grant sponsor: Carrillo-Oñativia grant (Ministerio de Salud), Argentina.

N.M. Giusto is a research member of the CONICET.

*Correspondence to: Instituto de Investigaciones Bioquímicas, Universidad Nacional del Sur y CONICET, C.C. 857, B8000FWB Bahía Blanca, Argentina. E-mail: ngiusto@criba.edu.ar

Received 14 March 2002; Revised 31 May 2002; Accepted 3 June 2002 
1956) or by exchange with the base moiety of preexisting glycerophospholipids (Porcellati et al., 1971; Kanfer and McCartney, 1991). A third pathway involves the production of phosphatidylserine (PS) from free serine by baseexchange, and its decarboxylation (Vance, 1998). This decarboxylation is catalyzed by the mitochondrial enzyme phosphatidylserine decarboxylase(PSDC) (EC 4.1.1.65).

A minimal value of $7 \%$ for the contribution of PSDC to the whole brain PE synthesis has been reported (Butler and Morell, 1983); however, the metabolic significance of PSDC in the brain is not clear. In this paper, we study the effect of aging on PSDC activity. To this end we assayed PSDC activity in the cerebral cortex (CC) and cerebellum (CRBL) mitochondrial fraction from adult (4-month-old) and aged (30-month-old) rats.

Recent reports demonstrate that hydrophilic PS species are decarboxylated by PSDC much faster than more hydrophobic ones in cell cultures, and that there is a substrate tissue-dependent preference (Heikinheimo and Somerharju, 1998; Kevala and Kim, 2001). In the present study, substrates with different docosahexaenoic acid (22: 6n-3) content were used to evaluate whether PSDC shows any substrate preference and if so, whether this preference undergoes changes during aging. The results obtained in $\mathrm{CC}$ and $\mathrm{CRBL}$ mitochondrial fraction were compared to those obtained in liver to establish whether the differences observed in CNS were tissue-specific.

\section{MATERIALS AND METHODS}

Wistar strain rats were kept under constant environmental conditions and fed on a standard pellet diet. $\mathrm{L}-\left[\mathrm{G}-{ }^{3} \mathrm{H}\right]$ serine (specific activity $21.7 \mathrm{Ci} / \mathrm{mmol}$ ) and Omnifluor were obtained from New England Nuclear-Dupont (Boston, MA). Triton X-100 was obtained from Sigma-Aldrich, (St. Louis, MO). All other chemicals were of the highest purity available.

\section{Preparation of Subcellular Fractions}

Homogenates were prepared from the CC, CRBL and liver of adult and aged rats. Rats were killed by decapitation and all brain areas and liver were immediately dissected $(2-4 \mathrm{~min}$ after decapitation).

CC and CRBL homogenates were prepared in the following way: $20 \%(\mathrm{w} / \mathrm{v})$ in $0.32 \mathrm{M}$ sucrose, $1 \mathrm{mM}$ EDTA, $5 \mathrm{mM}$ buffer HEPES ( $\mathrm{pH}$ 7.4). The homogenate was centrifuged at $1,300 \times g$ for $3 \mathrm{~min}$, and the supernatant was carefully poured into another tube. The nuclear pellet was resuspended with the isolation medium and spun at $1,300 \times g$ for $3 \mathrm{~min}$. The combined supernatant was subsequently centrifuged at 17,000 $\times$ $g$ for 10 min to obtain the crude mitochondrial pellet (CM), which was resuspended in the isolation medium and layered onto a 2-step gradient of 7.5-13\% Ficoll solution prepared in the isolation medium (Lai and Clark, 1979). The sample layered onto Ficoll discontinuous gradient was centrifuged at 99,000 $\times$ $g$ for $30 \mathrm{~min}$ in a swinging bucket rotor. The myelin fraction band was at the interface between the isolation medium and the 7.5\% Ficoll medium; the synaptosomal fraction (Syn) band at the interface between the $7.5 \%$ and the $13 \%$ Ficoll medium; and the purified mitochondrial fraction was the pellet below the $13 \%$ Ficoll medium (Lai and Clark, 1979).
Liver homogenates were prepared in the following way: $20 \%(\mathrm{w} / \mathrm{v}$ ) in $0.25 \mathrm{M}$ sucrose, $1 \mathrm{mM}$ EDTA, $5 \mathrm{mM}$ buffer HEPES ( $\mathrm{pH}$ 7.5). The liver homogenate was filtered through a 100-mesh Nitex, centrifuged at $960 \times g$ for $5 \mathrm{~min}$ and the supernatant filtered again and carefully poured into another tube. The nuclear pellet was resuspended with the isolation medium and spun at $960 \times g$ for $5 \mathrm{~min}$. The combined supernatant was subsequently centrifuged at $17,000 \times g$ for $10 \mathrm{~min}$ to obtain the CM pellet. To wash the mitochondrial pellet, it was resuspended in the isolation medium, then centrifuged at $17,000 \times g$ for $10 \mathrm{~min}$.

\section{Preparation of Radioactive Phosphatidylserine}

Radioactive PS, which was used as substrate, was obtained from bovine retinas and rat brain microsomal membranes. These membranes were incubated with $\mathrm{L}-\left[\mathrm{G}-{ }^{3} \mathrm{H}\right]$ serine under conditions favoring a base-exchange reaction yielding labeled PS (Ilincheta de Boschero et.al., 2000a).

Bovine eyes were obtained from a local abattoir and placed on ice within $10 \mathrm{~min}$ of the death of the animal. Retinas were dissected from the eyes and kept in a container with ice. After dissection, the retinas were incubated with $\left[{ }^{3} \mathrm{H}\right]$ serine (40 $\mu \mathrm{Ci} /$ retina) in Ames-Hastings medium in the presence of $500 \mu \mathrm{M}$ propranol at $37^{\circ} \mathrm{C}$ during $90 \mathrm{~min}$ (Ames and Hastings, 1956; Ilincheta et al., 1980). Lipids were extracted from the tissue as described by Folch et al. (1957). The extract containing total phospholipids was separated by TLC using silica gel $\mathrm{H}$ plate and a mobile phase consisting of chloroform/methanol/ ammonia (65:25:5 by volume) (Rouser et al., 1970).

$\left[{ }^{3} \mathrm{H}\right]$ Phosphatidylserine was scraped off the plate, eluted (Arvidson, 1968), and purified by a second TLC using silica gel $\mathrm{H}$ in acetone/chloroform/methanol/acetic acid/water (40:30: 10:10:4 by volume) as system (Rouser et al., 1970). The purified substrate was stored at $-30^{\circ} \mathrm{C}$ in chloroform/methanol (2:1 by volume) under nitrogen.

CC from adult and aged rats was immediately dissected after rat decapitation. CC homogenates were processed as described above to obtain the CM, which, was used as the starting material for obtaining purified mitochondrial fraction. The postmitochondrial supernatant was centrifuged at $105,000 \times g$ for $60 \mathrm{~min}$, to obtain CC microsomal fraction. Brain microsomal membranes from adult and aged rats were incubated with $\left[{ }^{3} \mathrm{H}\right]$ serine. Briefly, $10 \mu \mathrm{Ci}$ of $\left[{ }^{3} \mathrm{H}\right]$ serine were added to $1 \mathrm{ml}$ of microsomal membranes (2 $\mathrm{mg}$ protein) in $50 \mathrm{mM}$ HEPES, $6 \mathrm{mM} \mathrm{CaCl}_{2}, \mathrm{pH} 8.0$ and were incubated at $37^{\circ} \mathrm{C}$ for $30 \mathrm{~min}$. $\left[{ }^{3} \mathrm{H}\right]$ Phosphatidylserine was obtained from the tissue as described above.

Radioactivity and phosphorus content were measured for the determination of $\left[{ }^{3} \mathrm{H}\right] \mathrm{PS}$ specific activities. $\left[{ }^{3} \mathrm{H}\right]$ Phosphatidylserine obtained from bovine retina (rPS) had a specific radioactivity of $800 \mathrm{dpm} / \mathrm{nmol}$ whereas in $\left[{ }^{3} \mathrm{H}\right]$ phosphatidylserine obtained from adult (adPS) and aged (agPS) rat brain microsomal membranes, the specific radioactivity was 2,000 and 2,300 dpm/ nmol, respectively. Fatty acid methyl esters were analyzed (prepared by BF3-catalyzed methanolysis) by gas-liquid chromatography to determine 22:6 n-3 content from rPS, adPS and agPS (Aveldaño and Bazán, 1983; Lopez and Giusto, unpublished results) (Table I). 
TABLE I. Docosahexaenoic Acid Content in Phosphatidylserine Used as PSDC Substrate ${ }^{\star}$

\begin{tabular}{|c|c|c|}
\hline Source & Substrate & $22: 6 \mathrm{n}-3$ content $(\mathrm{mol} \%)$ \\
\hline Bovine retina & rPS & 36 \\
\hline Adult CC microsomes & adPS & 25 \\
\hline Aged CC microsomes & agPS & 21 \\
\hline
\end{tabular}

$\star$ Phosphatidylserine was obtained from different sources in order to provide PS with different 22:6n-3 content. Bovine retina PS (rPS) 22:6n-3 content determined by Aveldaño and Bazán (1983). Adult CC microsomal PS (adPS) and aged CC microsomal PS (agPS) 22:6n-3 content was determined by Lopez and Giusto, (unpublished results).

\section{Determination of PSDC Activity}

PSDC activity was assayed in vitro by measuring the production of radioactive PE from $\left[{ }^{3} \mathrm{H}\right] \mathrm{PS}$ labeled with $\mathrm{L}-[\mathrm{G}-$ $3 \mathrm{H}]$ serine according to Butler and Morell (1983). Briefly, $50 \mu \mathrm{l}$ of lipid vesicles containing $\left[{ }^{3} \mathrm{H}\right] \mathrm{PS}$ to yield $25 \mathrm{nmol} /$ assay were added to $100 \mu \mathrm{l}$ of mitochondrial membranes (200 $\mu \mathrm{g}$ protein) in a total volume of $200 \mu \mathrm{l}$ in buffer containing $100 \mathrm{mM}$ potassium phosphate ( $\mathrm{pH} 7.0), 1 \mathrm{mM}$ EDTA and $0.05 \%$ Triton X-100.

Lipid vesicles were prepared by drying the PS extract under nitrogen stream and resuspended by sonication at $25^{\circ} \mathrm{C}$ for $10 \mathrm{~min}$ in buffer solution. The reaction was incubated at $37^{\circ} \mathrm{C}$ for $30 \mathrm{~min}$ and stopped by the addition of $5 \mathrm{ml}$ of chloroform/methanol, (2:1 by volume). The lipids were extracted and separated as described below. The PE formation was quantified by liquid scintillation spectroscopy.

\section{Extraction and Isolation of Lipids}

Lipids were extracted according to Folch et al. (1957). Briefly, the lipid extract was washed with 0.2 volumes of $\mathrm{KCl}$ $(0.1 \mathrm{M})$ and the lower phase was obtained after centrifugation at 3,000 rpm for $5 \mathrm{~min}$. The phospholipids were then separated by one-dimensional TLC using silica gel G precoated plates (Merck) in a mobile phase consisting of chloroform/methanol/ ammonia (65:25:5 by volume) (Butler and Morell, 1983). The phospholipids were visualized by exposure of the plate to iodine vapors.

\section{Other Methods}

Protein and lipid phosphorus were determined according to Bradford et al. (1976) and Rouser et al. (1970), respectively.

\section{Statistical Analysis}

Statistical analysis was carried out using two-way ANOVA (age $X$ substrate). Significant differences were described using Roman small letters in the figures, with the values representing the mean $\pm \mathrm{SD}$ of 6 individual samples from different animals.

\section{RESULTS}

In this work we studied the synthesis of PE through the decarboxylation of PS in the CC and CRBL mitochondrial fraction from adult and aged rats. Both the behavior of PSDC during aging and the enzyme activity in the presence of substrates from different sources with

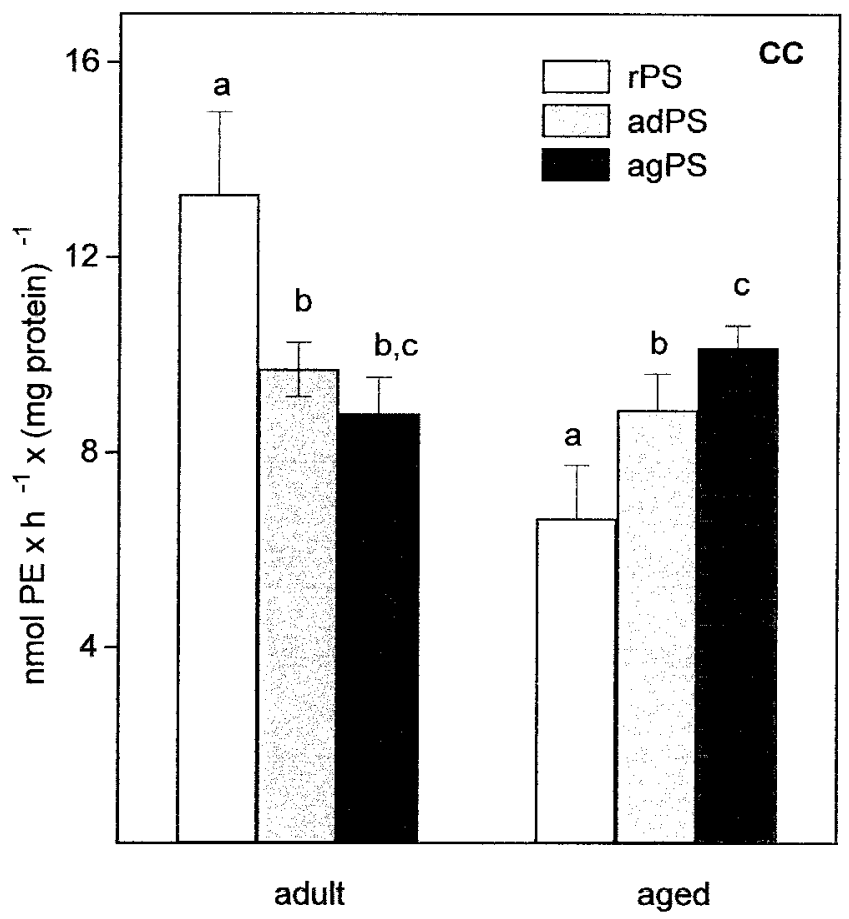

Fig. 1. PSDC activity in cerebral cortex mitochondrial fraction from adult and aged rats. PSDC activity was determined in purified CC mitochondrial fraction from adult and aged rats, by measuring phosphatidylethanolamine (PE) production as described in Materials and Methods. The enzyme activity was evaluated using as substrate phosphatidylserine (PS) from different sources: rPS ; adPS; and agPS. Enzyme activity is expressed as (nmol PE) $\times \mathrm{h}^{-1} \times$ (mg protein $)^{-1}$. Data are the mean $\pm \mathrm{SD}$ of 6 individual samples per condition. Each sample was obtained from a different adult (4-month-old) or aged (30-monthold) animal. Two-way ANOVA test (age $\times$ substrate) detected significant interaction (VI) in CC $(\alpha<0.05)$.

different fatty acid composition were evaluated. To this end PS was synthesized by $\left[{ }^{3} \mathrm{H}\right]$ serine incubation with bovine retina (rPS), adult rat CC microsomes (adPS), or aged rat CC microsomes (agPS), giving rise to 3 different kinds of substrate with different 22:6n-3 contents for the evaluation of PSDC activity (Table I).

\section{PSDC Activity in CC Mitochondrial Fraction From Adult and Aged Rats}

Figure 1 shows PSDC activity in CC mitochondrial fraction from adult and aged rats using as substrate PS synthesized from: a) bovine retina (36 mol\% $22: 6 n-3)$ or b) adult or aged CC microsomes (25 and 21 mol\% 22:6n-3 respectively). PSDC activity in adult CC mitochondria showed no changes in the presence of adPS or agPS; the highest activity was with rPS, demonstrating that substrates with a high 22:6n-3 content stimulates adult CC PSDC activity. Interestingly, PSDC activity in aged mitochondrial membranes increased by $16 \%$ in the presence of agPS compared to that in mitochondria from adult rats. Furthermore, PSDC activity in aged membranes in the 


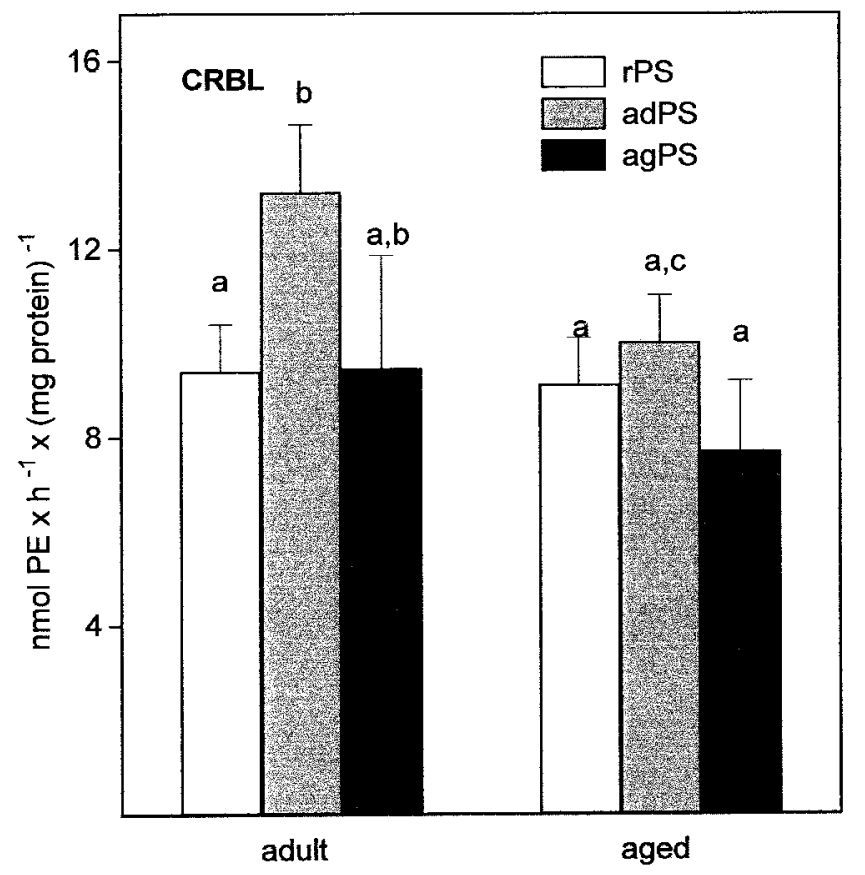

Fig. 2. PSDC activity in cerebellum mitochondrial fraction from adult and aged rats. PSDC was assayed as described in Figure 1 using as enzymatic source purified cerebellar mitochondrial fraction from adult and aged rats. Enzyme activity is expressed as (nmol PE) $\times \mathrm{h}^{-1} \times(\mathrm{mg}$ protein) ${ }^{-1}$. Data are the mean $\pm \mathrm{SD}$ of 6 individual samples per condition. Each sample was obtained from a different adult (4-monthold) or aged (30-month-old) animal. Two-way ANOVA test (age $\times$ substrate) did not detect significant interaction (VI) in CC $(\alpha<0.05)$.

presence of adPS showed $17 \%$ inhibition with respect to the activity observed in the presence of agPS. This result could indicate a certain degree of adaptability of the enzyme to its physiological substrate.

When PSDC was assayed in CC mitochondrial membranes from adult and aged rats in the presence of rPS, the activity of the enzyme in the latter decreased by $50 \%$ with respect to that in the former. These results show that a substrate enriched in 22:6n-3 provokes a high inhibition on mitochondrial PSDC activity from aged rats (Fig. 1).

\section{PSDC Activity in CRBL Mitochondrial Fraction From Adult and Aged Rats}

CRBL PSDC activity showed a different behavior from that of CC mitochondrial PSDC. PSDC from adult mitochondrial membranes showed the highest activity in the presence of adPS, 30\% higher than in membranes from aged rats. Using agPS as substrate, the lowest activity was found in mitochondrial membranes from aged rats, whereas PSDC from adult rats showed no changes with respect to the values found with adPS (Fig. 2). Interestingly, PSDC activity levels were the same in adult and aged membranes when rPS was used as substrate (Fig. 2).

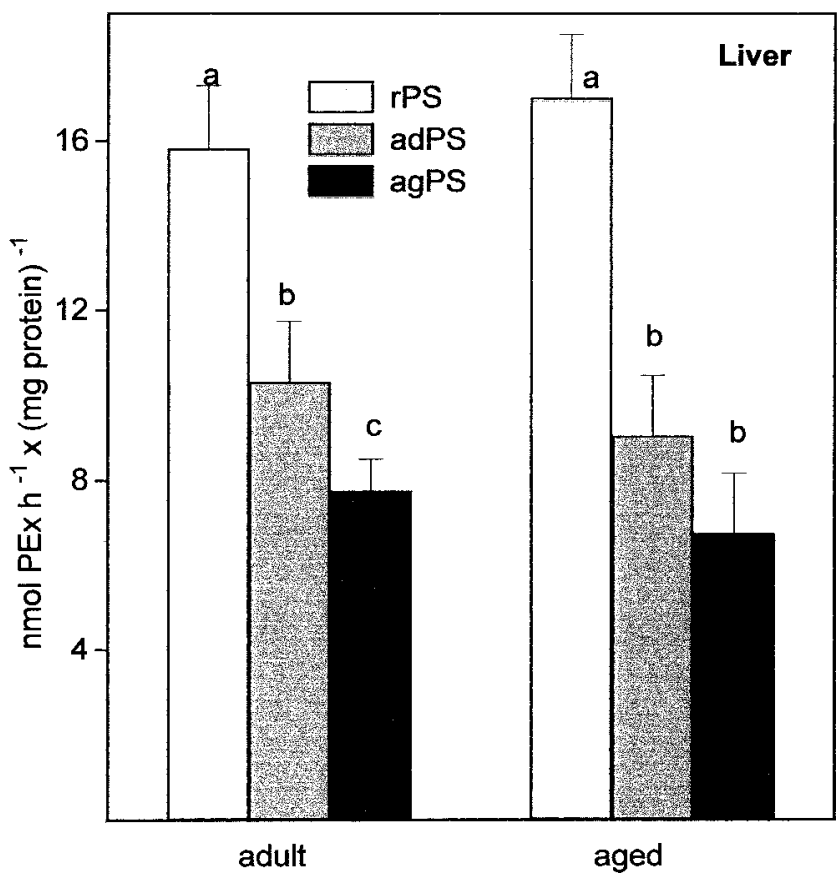

Fig. 3. PSDC activity in liver crude mitochondrial fraction from adult and aged rats. PSDC was assayed as described in Figure 1, using as enzymatic source, liver mitochondrial fraction. Enzyme activity is expressed as in Figure 1, data are the mean \pm SD of 6 individual samples per condition. Each sample was obtained from a different adult or aged animal (ages as in Fig. 1). Two-way ANOVA test did not detect significant interaction (VI) in CC $(\alpha<0.05)$.

\section{PDSC Activity in Liver Mitochondrial Fraction From Adult and Aged Rats}

To determine whether or not the changes observed in PSDC activity during aging correspond exclusively to the CNS, similar assays were carried out in liver mitochondrial membranes from both adult and aged rats. PSDC showed higher activity in the presence of adPs (35\% increase) in adult membranes than in the presence of agPS in aged membranes. When liver mitochondria from adult rats were incubated with agPS, PE biosynthesis was inhibited by $28 \%$ with respect to the activity found in the presence of adPS (Fig. 3). PSDC activity showed no changes in adult or aged mitochondrial membranes in the presence of rPS. Under the same experimental conditions, however, liver PSDC showed the highest activity compared to the values found in the presence of adPS or agPS (Fig. 3).

In Figure 4A,B results are expressed in terms of ratios comparing PSDC activity in CC or CRBL to that in liver. In CC, the most striking ratios were found in aged animals in the presence of agPS (1.5) and in the presence of rPS (0.3). These values corroborate the fact that in aged CC the most favorable substrate is aged microsomal PS, whereas PS with a high 22:6n-3 content inhibits PSDC activity with respect to the values found in liver (Fig. 4A). 
Fig. 4. Relationship between CNS and liver PSDC activity in adult and aged rats. A: PSDC activity levels in CC mitochondrial fraction were compared to enzyme levels found in mitochondrial fraction from liver under the same experimental conditions. Data are expressed as the ratio of PSDC in CC with respect to liver in adult and aged rats. B: PSDC activity levels in cerebellar mitochondrial fraction were compared with enzyme levels found in mitochondrial fraction from liver. Data are expressed as the ratio of PSDC in cerebellum with respect to liver in adult and aged rats.

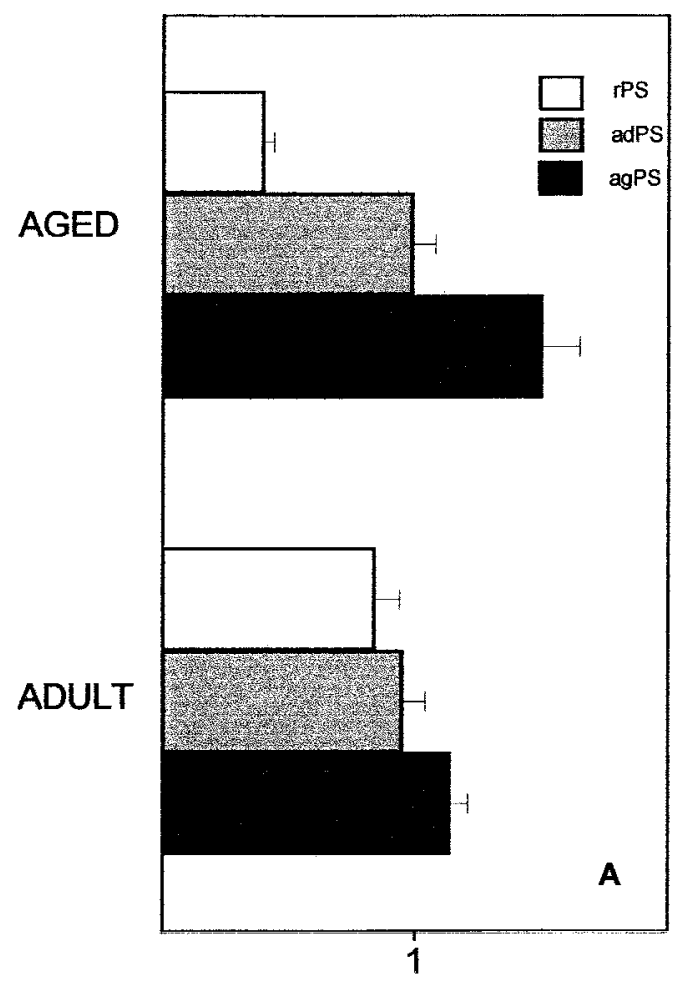

CC PSDC / Liver PSDC

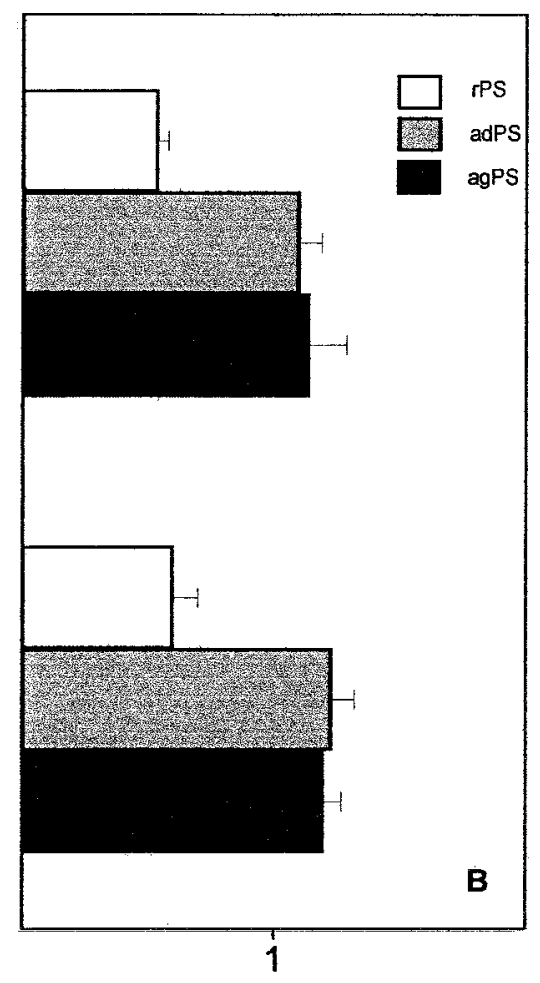

CRBL PSDC / Liver PSDC
In CRBL the highest ratio was registered in adult membranes in the presence of adPS (1.41). These results demonstrate that PSDC from liver is less active in adults in the presence of adult microsomal PS, whereas in aged membranes, liver PSDC is more active than CRBL PSDC in the presence of agPS. The ratios in adult and aged membranes were 0.65 and 0.56 respectively, in the presence of rPS; demonstrating that the retinal substrate was better decarboxylated in liver than in CRBL for both age groups. (Fig. 4A).

\section{DISCUSSION}

We report evidence that PSDC from CC and CRBL mitochondrial fraction behaves differently during aging.

To evaluate the substrate specificity of PSDC in mitochondrial membranes from adult and aged rats, three PS classes obtained from different sources were used in our assays. One source was bovine retina, in which this phospholipid has a high 22:6n-3 content (36 mol\%) (Aveldaño and Bazán, 1983), and the other two CC microsomes from adult and aged rats. Previous studies in our laboratory demonstrate that PS composition changes in microsomal membranes during aging. The content of $22: 6 n-3$ esterified to PS decreases by $17 \%$ in agPS (21 mol\%) with respect to adPS (25 mol\%), whereas oleic acid content in this phospholipid increases by the same magnitude (Lopez and Giusto, unpublished results) (Table I).

In CC, PSDC activity showed no changes when adult membranes were assayed with adPS and aged mem- branes with agPS. These results demonstrate that in the presence of its physiological substrate, PSDC, does not undergo changes during aging. In the presence of agPS however, PSDC from adult membranes was inhibited by $16 \%$ with respect to the activity found in aged membranes. The inhibition (13\%) of PSDC activity in aged rats in the presence of adPS with respect to the values found in the presence of its physiological substrate (agPS) may suggest that during aging PSDC shows a preference for substrates with a fatty acid composition that is low in 22:6n-3 content. This is corroborated by the fact that when rPS (high 22:6n-3 content) was used, PSDC from aged rats was inhibited by $50 \%$ with respect to the adult values and $36 \%$ with respect to the activity found in the presence of agPS in aged membranes.

Taking into account the fact that microsomal PS fatty acid composition undergoes changes during aging and that mitochondrial CC PSDC in aged rats is more active in the presence of its physiological substrate (agPS), it can be concluded that the enzyme adapts itself to using the available substrate to maintain its activity.

It has been demonstrated that PS is presented to mitochondria through the mitochondria-associated membrane (MAM), where PS can be synthesized (Voelker, 1985, 1997, 2000; Shiao et al., 1995). In addition, PS hydrophilic species $(18: 0,22: 6 n-3)$ are transported and decarboxylated more efficiently than hydrophobic ones (Nichols, 1988; Ponwall et al., 1991; Heikinheimo and Som- 
erharju, 1998). These findings could explain the levels of PSDC activity in adult rats, indicating that rPS is readily incorporated into the $\mathrm{CC}$ mitochondrial membrane.

Kevala and Kim (2001) constructed liposomes with 3 different PS species and demonstrated that 18:0,22:6n-3 species are more efficiently decarboxylated in brain cortex. They also suggested that the observed substrate preference in CNS could not be due to the characteristics of substrate presentation, but to the specificity of the enzyme. In agreement with this finding, higher PSDC activity in adult rats was observed when rPS was used as substrate; however, the inhibition of mitochondrial CC PSDC activity in aged rats by PS species with a high 22:6n-3 content (rPS) could be due to a change of substrate specificity during aging rather than to a transport phenomenon.

In CRBL mitochondrial fraction from adult rats, PSDC underwent no changes in the presence of alternative substrates (adPS or agPS). These findings are similar to those obtained with CC in adult animals; however, in aged membranes PSDC showed lower activity levels than those in adult membranes under the same experimental conditions. The lowest activity was observed in mitochondrial fraction from aged rats in the presence of agPS as substrate; PSDC under these conditions was $47 \%$ inhibited with respect to PSDC from adult rats assayed with adPS.

In the presence of adPS, senile cerebellar PSDC activity is lower than the activity found in adult membranes. The fact that rPS does not produce change in PSDC activity from aged membranes with respect to the values in adult membranes implies cerebellar PSDC activity is not modulated by the 22:6n-3 content of the substrate. Our results demonstrate that PSDC behavior in $\mathrm{CRBL}$ is quite different from that in CC, suggesting that aging exerts a different modulation on cerebellar PSDC.

Using adPS and agPS, enzyme activity levels in liver were the same in adult and aged membranes. In the presence of agPS, however, the activity levels at both ages (4 and 30months old) were 25-35\% lower than in the presence of adPS. When rPS was presented as substrate, liver PSDC did not change during aging. Furthermore, the activity levels found in liver mitochondrial fraction in the presence of a substrate rich in 22:6n-3 were higher than the levels found in the presence of adPS or agPS. These results demonstrate that liver PSDC has a clear dependence on PS 22:6n-3 content.

Our assays on liver show that PSDC behavior is different from that reported for CNS during aging. Activity ratios for CC and CRBL and the activities found in liver with the different PSs permit us to conclude that substrate selectivity in CNS is different from that in liver. In $\mathrm{CC}$ and $\mathrm{CRBL}$ the lowest ratios were found in aged mitochondrial fraction in the presence of rPS. Regarding these findings, it can be concluded that PSDC activity during aging is strongly inhibited with respect to the values found in liver in the presence of rPS. This demonstrates that during aging, CNS PSDC changes its substrate preference with respect to liver. (Fig. 4A,B).
Phosphatidylethanolamine is one of the most abundant phospholipids in eukaryotic cells (de Kruijff, 1997). Membranes with high PE content can undergo laminarhexagonal transition affecting integration of proteins to membranes, lateral movement in the membrane and folding and stabilization of certain protein complexes. Specific PE and PS molecular species profiles in cell membranes are likely due to the molecular specificity involved in various biosynthetic and remodeling processes including PS decarboxylation. In the brain, PS and PE are the phospholipids most enriched in 22:6n-3 and the most susceptible to a decrease in 22:6n-3 content both in CC and CRBL during dietary depletion of $\mathrm{n}-3$ fatty acids or during aging (Salem et al., 1986; Lopez et al., 1995; Hamilton et al., 2000). Our results show that during aging the behavior of PSDC in CC is different from that in CRBL. It can be concluded that in CC, substrate specificity of PSDC in aged rats correlates with the composition of $\mathrm{PE}$ in aged CC membranes, thus suggesting that PSDC contributes significantly to membrane lipid composition. It can also be concluded that in aged CRBL, the PE profile is determined by individual phospholipid biosynthetic and remodeling processes that are responsible for PE composition in this CNS region.

In summary, the current data indicate that substrate preference of PSDC, based on 22:6n-3 content, changes during aging and that this preference is organ-specific. These findings provide a starting point for further studies aimed at understanding the physiological role of PSDC in CNS during aging.

\section{REFERENCES}

Ames A III, Hastings B. 1956. Studies on water and electrolytes in nervous tissue, I. rabbit retina: methods and interpretation of data. J Neurophysiol 19:201-212.

Arvidson GAE. 1968. Structural and metabolic heterogeneity of rat liver glycerophosphatidates. Eur J Biochem 4:478-486.

Aveldaño MI, Bazán NG. 1983. Molecular species of phosphatidylcholineethanolamine, -serine and -inositol in microsomal and photoreceptor membranes of bovine retina. J Lipid Res 24:620-627.

Bradford MM. 1976. A rapid and sensitive method for the quantitation of microgram quantities of protein utilizing the principle of protein-dye binding. Anal Biochem 72:248-254.

Butler M, Morell P. 1983. The role of phosphatidylserine decarboxylase in brain phospholipid metabolism. J Neurochem 41:1445-1450.

de Kruijff B. 1997. Lipid polymorphism and biomembrane function. Curr Opin Chem Biol 1:564-569.

Folch J, Lees M, Sloane Stanley GH. 1957. A simple method for the isolation and purification of total lipids from animal tissues. J Biol Chem 226:497-509.

Giusto NM, Roque ME, Ilincheta de Boschero M. 1992. Effects of aging on the content, composition and synthesis of sphingomyelin in the central nervous system. Lipids 27:835-839.

Hamilton L, Greiner R, Salem N Jr, Kim HY. 2000. n-3 fatty acids deficiency decreases phosphatidylserine accumulation selectively in neuronal tissues. Lipids 35:863-869.

Harman D. 1991. The aging process: major risk factor for disease and death. Proc Natl Acad Sci USA 88:5360-5363.

Heikinheimo L, Somerharju P. 1998. Preferential decarboxylation of hydrophilic phosphatidylserine species in cultured cells. J Biol Chem 273: 3327-3335. 
Ilincheta de Boschero MG, Giusto NM, Bazán NG. 1980. Biosynthesis of membrane lipids in the retina: subcellular distribution and propranolol action on phosphatidic acid, phosphatidylserine and phosphatidylethanolamine. Neurochemistry 1:17-28.

Ilincheta de Boschero MG, Roque ME, Salvador GA, Giusto NM. 2000a. Alternative pathways for phospholipid synthesis in different brain areas during aging. Exp Gerontol 35:653-668.

Ilincheta de Boschero MG, López GH, Castagnet PI, Giusto NM. 2000b. Differential incorporation of precursor moieties into cerebral cortex and cerebellum glycerophospholipids during aging. Neurochem Res 25:875884.

Kanfer JN, McCartney D. 1991. Sphingosine and unsaturated fatty acids modulate the base exchange enzyme activities of rat brain microsomes. FEBS Lett 291:63-66.

Kennedy EP, Weiss SB. 1956. The function of cytidine coenzymes in the biosynthesis of phospholipids. J Biol Chem 222:193-214.

Kevala JH, Kim HY. 2001. Determination of substrate preference in phosphatidylserine decarboxylation by liquid chromatographyelectrospray ionization mass spectrometry. Anal Biochem 292:130-138.

Lai JCK, Clark JB. 1979. Preparation of synaptic and nonsynaptic mitochondria from mammalian brain. Methods Enzymol LV:51-60.

López GH, Ilincheta de Boschero MG, Castagnet PI, Giusto NM. 1995. Age-associated changes in the content and fatty acid composition of brain glycerophospholipids. Comp Biochem Physiol 112:331-343.

Lynch RD. 1980. Utilization of polyunsaturated fatty acids by human diploid cells aging in vitro. Lipids 15:412-420.

Nichols JW. 1988. Phospholipid transfer between phosphatidylcholinetaurocholate mixed micelles. Biochemistry 27:3925-3931

Pasquaré SJ, Ilincheta de Boschero MG, Giusto NM. 2001. Aging promotes a different phosphatidic acid utilization in cytosolic and microsomal fractions from brain and liver. Exp Gerontology 36:1387-1401.

Ponwall HJ, Bick DL, Massey JB. 1991. Spontaneous phospholipid transfer: development of a quantitative model. Biochemistry 30:5696-5700.

Porcellati G, Arienti G, Pirotta A, Giorgini D. 1971. Base exchange reactions for the synthesis of phospholipids in nervous tissue: the incorporation of serine end ethanolamine into the phospholipids of isolated brain microsomes. J Neurochem 18:1395-1417

Rouser G, Fleischer S, Yamamoto A. 1970. Two dimensional thin layer chromatographic separation of polar lipids and determination of phospholipids by phosphorus analysis of spots. Lipids 5:494-496.

Salvador GA, Pasquaré SJ, Ilincheta de Boschero MG, Giusto NM. 2002. Differential modulation of phospholipase D and phosphatidate phosphohydrolase during aging in rat cerebral cortex synaptosomes. Exp Gerontol 37:543-552.

Salem N Jr, Kim H-Y, Yergey JA. 1986. Docosahexaenoic acid membrane function and metabolism. In: Simopoulos A, Martin R, Kifer R, editors. Health effects of polyunsaturated fatty acids in seafood. New York: Academic Press. p 263-317.

Shiao YJ, Lupo G, Vance JE. 1995. Evidence that phosphatidylserine is imported into the mitochondria via a mitochondria-associated membrane and that the majority of phosphatidylethanolamine is derived from decarboxylation of phosphatidylserine. J Biol Chem 270:11190-11198.

Stubbs CD, Smith AD. 1984. The modification of mammalian membrane polyunsaturated fatty acid composition in relation to membrane fluidity and function. Biochim Biophys Acta 779:89-137.

Vance JE. 1998. Eukaryotic lipid-biosynthetic enzymes: the same but not the same. Trends Biochem Sci 23:423-428.

Voelker DR. 1985. Disruption of phosphatidylserine translocation to the mitochondria in baby hamster kidney cells. J Biol Chem 260:1467114676.

Voelker DR. 1997. Phosphatidylserine decarboxylase. Biochim Biophys Acta 1348:236-244.

Voelker DR. 2000. Interorganelle transport of aminoglycerophospholipids. Biochim Biophys Acta 1486:97-107.

Yen TZ, King KL, Lee HC, Yeh SH, Wei YH. 1994. Age dependent increase of mitochondrial DNA deletions together with lipid peroxides and superoxide dismutase in human liver mitochondria. Free Radic Biol Med 16:207-214. 